\title{
The colour-lightcurve shape relation of type la supernovae and the reddening law
}

\author{
S. Nobili and A. Goobar
}

\author{
Department of Physics, Stockholm University, 10691 Stockholm, Sweden \\ e-mail: serena@physto.se
}

Received 20 December 2007 / Accepted 4 April 2008

\section{ABSTRACT}

\begin{abstract}
Aims. We present a study of the time sequence of optical colours of a large sample of nearby type Ia supernovae (SNe Ia). We study the dependence of the colour time-evolution on the lightcurve shape, parametrized by the stretch factor.

Methods. We determine the spectral template that minimizes the dispersion in the colours of SNe Ia, measured by UBVRI photometry of nearby supernovae.

Results. A clear colour dependence upon lightcurve shape is found, such that the narrower lightcurves are redder up to about one month past lightcurve maximum. After correcting for lightcurve-shape differences in intrinsic colour, we derive an average reddening law, that is well described by a Cardelli, Clayton \& Mathis law with $R_{V}=1.75 \pm 0.27$ for 80 type Ia supernovae with $E(B-V) \leq 0.7$ mag. A subset of $69 \mathrm{SNe}$ that have modest reddening, $E(B-V)<0.25 \mathrm{mag}$, gives a significantly smaller value, $R_{V} \sim 1$, which suggests that the observed reddening of type Ia supernovae may have a more complex origin, perhaps associated with other processes besides extinction by interstellar dust inside the host galaxy.
\end{abstract}

Key words. stars: supernovae: general - ISM: dust, extinction - methods: statistical

\section{Introduction}

Type Ia supernovae (SNe Ia) have been successfully utilized as standard candles in cosmology, which have helped to probe the acceleration of the Universe by the as yet undetermined means of dark energy (Riess et al. 1998; Perlmutter et al. 1999). More recently, we observed the beginning of the second generation of supernova surveys, wich involve dedicated supernova searches (e.g. $\mathrm{SNLS}^{1}, \mathrm{SDSS}^{2}, \mathrm{ESSENCE}^{3}$ ) and massive spectroscopic follow-up. The first results from these surveys indicated that simply increasing the number of supernovae that populate the Hubble diagram at high redshift, may be insufficient to discriminate among different dark energy models. Systematic uncertainties were found to be the limiting factors of ongoing supernova projects (Astier et al. 2006; Wood-Vasey et al. 2007).

Dimming of light by dust along the line-of-sight, predominantly in the host galaxy of the supernova explosion, is one the main sources of systematic uncertainty, see e.g. Knop et al. (2003). For type Ia supernovae, two additional causes of reddening are sometimes suggested: extinction by circumstellar dust and the possibility of an intrinsic colour-brightness relation. These last two scenarios further complicate the prospects to disentangle all the potential effects and their possible evolution with cosmic time.

Our ability to correct for these effects relies on the knowledge of the intrinsic colours of SNe Ia, as well as on the nature of the reddening. The latter is often assumed to be due to Milky Way like dust extinction. We show in Sect. 6, however, that this is not necessarily the case and requires further investigation.

\footnotetext{
1 http://www. cfht.hawaii.edu/SNLS/

2 http://www.sdss.org/

${ }^{3}$ http://www.ctio.noao.edu/essence/
}

The effect of cosmological redshift on the measured broadband photometry is accounted for in a straight-forward manner by $K$-corrections (Kim et al. 1996). The supernova (SN) spectrum is usually not acquired for all epochs, however, since typically only few spectra are taken to allow type identification. In the standard technique, spectroscopic templates obtained by averaging spectra of well observed (mostly nearby) SNe Ia are used. Thus, the uncertainty in the $K$-corrections depends primarily on the spectroscopic diversity of SNe Ia. Nugent et al. (2002), however, showed that changes in individual spectral features do not significantly affect $K$-corrections, as long as there is a good match between the observed and the rest-frame bandpass filters, and they are instead mainly sensitive to the supernova colours. The knowledge of intrinsic colour of $\mathrm{SNe}$ can help to solve one of the limiting factors in supernova cosmology.

This issue becomes even more critical when dealing with observations of very high- $z \mathrm{SNe}$, for which the rest-frame extends, partly or entirely, into the UV part of the spectrum. Our limited knowledge of the supernova properties in the $U$-band, due to poor telescope and CCD sensitivity at these wavelengths, was identified as the main source of uncertainty in the determination of cosmological parameters in Knop et al. (2003).

Some efforts to observe $\mathrm{SNe}$ in the $U$-band have been made recently and a number of nearby $\mathrm{SN}$ data have been collected (Jha et al. 2006; Pastorello et al. 2007; Stanishev et al. 2007). There were also attempts to utilize high signal-to-noise spectra of more distant SN Ia from the SNLS sample, under the assumption that there is no evolutionary trend in the SN spectrum (Guy et al. 2005, 2007; Hsiao et al. 2007). This assumption may not however hold up to arbitrary high redshifts. Since the average metallicity of the universe increases with cosmic time, it is not unreasonable to expect that high redshift $\mathrm{SNe}$ Ia originate in environments of lower average metallicity. The effect on the 
spectral energy distribution of a lower metallicity progenitor was modelled by Hoeflich et al. (1998) and Lentz et al. (2000), who found that such SNe Ia, especially at early epochs, are expected to show enhanced flux in the $U V$ region of the spectrum, weaker absorption features in the optical, and a shift in the minima of optical absorption features to redder wavelengths (see, however, Ellis et al. 2007).

We present a statistical study of $U-B, B-V, V-R$, and $R-I$ colour evolution for 80 nearby supernovae. This work is a continuation of the analysis presented in Nobili et al. (2003), for an increased sample and with the addition of $U$-band data. This allowed us to study the dependence of colours on the stretch factor, which is, in turn, applied to correct the spectral templates used for computing the $K$-corrections. The knowledge of intrinsic colour dispersion is further used to study the average properties of the extragalactic reddening law of SNe Ia.

In Sect. 2, the data set used in this paper is presented. In Sect. 3, we investigate the correlation of colours with lightcurve shape, and estimate colour curve models of typical SNe Ia. The study of intrinsic dispersion in colours is reported Sect. 4. In Sect. 5, we discuss the use of our colour-stretch relation to modify the spectral template for computing $K$-corrections, and compare our results with previously published estimates. In Sect. 6, we finally discuss the implication of our analysis for computing the effective average extinction law of SNe Ia.

\section{The data set}

We present the analysis of the colour properties of a collection of 80 well-observed nearby $\mathrm{SNe}$ Ia for which data are available in the literature. This includes the Calan/Tololo data set (Hamuy et al. 1996), the CfA data set (Riess et al. 1998), which was analyzed in Nobili et al. (2003), the data set of Jha et al. (2006), and data of some single very well-observed nearby supernovae, i.e. SN 2001el (Krisciunas et al. 2003), SN 2000ca, and SN 2001ba (Krisciunas et al. 2004a), SN 2001cn (Krisciunas et al. 2004b), SN 2001V (Vinkó et al. 2003), SN 2002er (Pignata et al. 2004), SN 2003du (Stanishev et al. 2007), and SN 2005cf (Pastorello et al. 2007). Sub-luminous type Ia SNe, 1991bg-like, were not included nor were supernovae with poor $B$-band sampling about lightcurve maximum. Out of these $80 \mathrm{SNe}$, a subset with moderate colour excess, $E(B-V)<0.25 \mathrm{mag}^{4}$, was selected to study the intrinsic colours of type Ia supernovae. The complete list of the SNe used is reported in Table 1, with the observed filter data available, redshift, and fitted $B$-band stretch factor $(s)$ (Perlmutter et al. 1997; Goldhaber et al. 2001). Figure 1 shows the distribution of stretch factors and redshifts for the entire sample.

The data were $K$-corrected to the restframe bandpass, as defined by Kim et al. (1996), assuming Bessell-filter transmission curves (Bessell 1990). The spectral template used to compute the $K$-corrections was produced by Hsiao et al. (2007) by averaging about 600 spectra of SNe Ia at different epochs. Data considered in this analysis includes those of nearby supernovae with redshifts up to $\sim 0.1$, with most of the SNe at $z<0.06$ (see Fig. 1). Our ability to correct for extinction, i.e. to compute the colour excess $E(B-V)$, depends on our knowledge of the intrinsic colours of SNe Ia. An iterative procedure was therefore adopted until the procedure converged, as explained in the next section.

\footnotetext{
${ }^{4}$ In Sect. 6 we will relax this constrain, adding the remaining $11 \mathrm{SNe}$, and study the impact on the derived reddening law.
}

\section{The colour-stretch relation and colour time evolution}

The correlation between colours and lightcurve shape parameter is a known property of SNe Ia. Phillips et al. (1999) showed the dependence of $B-V$ and $V-I$ at lightcurve maximum on the shape parameter. We use a larger data set to extend this analysis to all epochs up to 60 days after lightcurve maximum. We extend the wavelength range that we study to include the $U$-band. As in Nobili et al. (2003), the SN colours are computed from the original data, without any interpolation. For this to be possible, the SN should have been observed in at least two different bands at the same epoch, which is often the case. Thus, the $B$-band lightcurve fit is performed exclusively with the aim of determining the time of maximum and the stretch factor. We consider the rest-frame time, since $B$-band maximum, $t$, and the stretch factor, $s$, as two independent variables. We introduce the colour excess $E(B-V)$ for each $\mathrm{SN}$ as independent variable. Each colour $U-B$, $B-V, V-R$ and $R-I$ is fitted with a parametrised function:

$X-Y=b_{X Y}(t)+a_{X Y}(t) \cdot(s-1)+c_{X Y} \cdot E(B-V)$

where $X$ and $Y$ are arbitrary filters, and $a(t)$ and $b(t)$ are determined using least-squares cubic-spline fit of 6 knots each, which are distributed in the time range between -10 and +60 days with respect to the time of maximum light. The knot positions are chosen to minimize the number of knots while yielding a good fit to the data. We note that, in this parametrisation, the $b$ functions correspond to the colour curves for a stretch factor $s=1 \mathrm{SN}$, while the $a$ functions provide the dependence of the colours on stretch. Different dependences on stretch were investigated, e.g. $\left(1 / s^{3}-1\right)$ used by Knop et al. (2003). The least complicated form of Eq. (1) provides the most appropriate description of the data. The $c_{X Y}$ parameters are fitted for each colour without assuming that the dimming of type Ia SNe has any specific wavelength dependence.

We applied the following iterative procedure:

1. fit the $B$-band lightcurve time of maximum and the stretch parameter, following Goldhaber et al. (2001);

2. compute the colour $U-B, B-V, V-R$, and $R-I$ for each $\mathrm{SN}$ at each observed epoch;

3. correct the observed colours for Milky-Way extinction, assuming the extinction law (Cardelli et al. 1989) (CCM), as modified by O'Donnell (1994). The corrections are computed by reddening the SNe Ia spectral template at different epochs, and integrating in all bandpasses;

4. compute $K$-corrections for each SN using the spectral template corrected by the colour-stretch relation determined in the previous iteration. In the first iteration, $K$-corrections were computed without using any colour-stretch relation, i.e. as if all SNe had $s=1$;

5. compute the colour excess $E(B-V)$ as the weighted-average excess, for all epochs, from the $B-V$ colour curve for each supernova determined in the previous iteration. The inverse square of the uncertainty on the individual colour measurements, $1 / \sigma_{i}^{2}$, is used as weights of each measurement when calculating the average, and the uncertainty on $E(B-V)$ is determined to be $1 / \sqrt{\sum 1 / \sigma_{i}^{2}}$. For the first iteration, an initial colour excess $E(B-V)$ for each SN was estimated following the method described by Phillips et al. (1999);

6. fit the function in Eq. (1) to the data separately for each colour;

7. modify the spectral template for the average colour-stretch relation. 
Table 1. List of SNe used for the analysis. References: (0), Wells et al. (1994); (1) Hamuy et al. (1996); (2) Riess et al. (1998); (3) Jha et al. (2006); (4) Krisciunas et al. (2003) (4a) Krisciunas et al. (2004a); (4b) Krisciunas et al. (2004b); (5) Vinkó et al. (2003); (6), Pignata et al. (2004); (7) Stanishev et al. (2007); (8), Pastorello et al. (2007).

\begin{tabular}{|c|c|c|c|c|c|c|}
\hline SN & Band & $z$ & $s$ & $E(B-V)^{M W}$ & $E(B-V)^{H G}$ & Ref. \\
\hline 1989B & $U B V R I$ & 0.004 & 0.904 & $0.032(0.003)$ & $0.464(0.021)$ & $(0)$ \\
\hline 19900 & $B V R I$ & 0.030 & 1.072 & $0.093(0.009)$ & $0.061(0.024)$ & (1) \\
\hline 1990T & $B V R I$ & 0.040 & 0.986 & $0.053(0.005)$ & $0.152(0.021)$ & (1) \\
\hline $1990 \mathrm{Y}$ & $B V R I$ & 0.039 & 1.252 & $0.008(0.001)$ & $0.488(0.026)$ & (1) \\
\hline 1991ag & $B V R I$ & 0.014 & 1.130 & $0.062(0.006)$ & $0.083(0.014)$ & (1) \\
\hline $1991 \mathrm{~S}$ & $B V R I$ & 0.056 & 0.945 & $0.026(0.003)$ & $0.024(0.021)$ & (1) \\
\hline $1991 U$ & $B V R I$ & 0.031 & 1.058 & $0.062(0.006)$ & $0.133(0.022)$ & (1) \\
\hline $1992 \mathrm{~A}$ & $B V R$ & 0.006 & 0.829 & $0.017(0.002)$ & $0.058(0.006)$ & (1) \\
\hline 1992ae & $B V$ & 0.075 & 0.914 & $0.036(0.004)$ & $0.096(0.024)$ & (1) \\
\hline 1992ag & $B V I$ & 0.026 & 0.820 & $0.097(0.010)$ & $0.267(0.017)$ & (1) \\
\hline 1992al & $B V R I$ & 0.014 & 0.922 & $0.034(0.003)$ & $-0.025(0.011)$ & (1) \\
\hline 1992au & $B V I$ & 0.061 & 0.769 & $0.017(0.002)$ & $0.130(0.034)$ & (1) \\
\hline $1992 b c$ & $B V R I$ & 0.020 & 1.089 & $0.022(0.002)$ & $-0.034(0.009)$ & (1) \\
\hline $1992 \mathrm{bg}$ & $B V I$ & 0.036 & 0.996 & $0.185(0.018)$ & $0.051(0.016)$ & (1) \\
\hline 1992bh & $B V I$ & 0.045 & 1.037 & $0.022(0.002)$ & $0.126(0.015)$ & (1) \\
\hline 1992bl & $B V I$ & 0.043 & 0.771 & $0.011(0.001)$ & $0.015(0.016)$ & (1) \\
\hline $1992 b p$ & $B V I$ & 0.079 & 0.853 & $0.069(0.007)$ & $-0.035(0.017)$ & (1) \\
\hline $1992 \mathrm{bs}$ & $B V$ & 0.063 & 0.958 & $0.012(0.001)$ & $0.043(0.015)$ & (1) \\
\hline 1992J & $B V I$ & 0.046 & 0.896 & $0.057(0.006)$ & $0.254(0.028)$ & (1) \\
\hline $1992 \mathrm{P}$ & $B V I$ & 0.026 & 1.084 & $0.021(0.002)$ & $0.036(0.017)$ & (1) \\
\hline $1993 \mathrm{ac}$ & $B V R I$ & 0.049 & 0.767 & $0.163(0.016)$ & $0.090(0.040)$ & (2) \\
\hline 1993ae & $B V R I$ & 0.019 & 0.932 & $0.039(0.004)$ & $0.071(0.013)$ & (2) \\
\hline 1993ag & $B V I$ & 0.050 & 0.909 & $0.112(0.011)$ & $0.132(0.019)$ & (1) \\
\hline 1993B & $B V I$ & 0.071 & 0.823 & $0.079(0.008)$ & $0.078(0.019)$ & (1) \\
\hline $1993 \mathrm{H}$ & $B V R I$ & 0.027 & 0.781 & $0.060(0.006)$ & $0.172(0.014)$ & (1) \\
\hline $1993 \mathrm{~L}$ & $B V R I$ & 0.005 & 1.159 & $0.014(0.005)$ & $0.457(0.030)$ & (1) \\
\hline 19930 & $B V I$ & 0.052 & 0.898 & $0.053(0.002)$ & $-0.012(0.013)$ & (1) \\
\hline 1994ae & $B V R I$ & 0.004 & 1.006 & $0.031(0.003)$ & $0.102(0.010)$ & (2) \\
\hline 1994D & $B V R I$ & 0.003 & 0.814 & $0.022(0.002)$ & $-0.063(0.000)$ & (2) \\
\hline 1994M & $B V R I$ & 0.023 & 0.800 & $0.024(0.002)$ & $0.093(0.015)$ & (2) \\
\hline 1994Q & $B V R I$ & 0.029 & 1.131 & $0.017(0.002)$ & $0.155(0.023)$ & (2) \\
\hline $1994 \mathrm{~S}$ & $B V R I$ & 0.015 & 1.023 & $0.021(0.002)$ & $0.027(0.019)$ & (2) \\
\hline 1994T & $B V R I$ & 0.035 & 0.928 & $0.029(0.003)$ & $0.061(0.021)$ & (2) \\
\hline $1995 \mathrm{ac}$ & $B V R I$ & 0.050 & 1.077 & $0.042(0.004)$ & $0.057(0.010)$ & (2) \\
\hline 1995ak & $B V R I$ & 0.023 & 0.831 & $0.043(0.004)$ & $0.109(0.021)$ & (2) \\
\hline 1995al & $B V R I$ & 0.005 & 1.061 & $0.014(0.001)$ & $0.169(0.013)$ & (2) \\
\hline 1995d & $B V R I$ & 0.007 & 1.069 & $0.058(0.006)$ & $0.042(0.011)$ & (2) \\
\hline 1996ab & $B V$ & 0.124 & 1.006 & $0.032(0.002)$ & $-0.024(0.023)$ & (3) \\
\hline 1996bk & $B V R I$ & 0.007 & 0.763 & $0.018(0.002)$ & $0.380(0.017)$ & (3) \\
\hline 1996bl & $B V R I$ & 0.036 & 0.982 & $0.105(0.011)$ & $0.077(0.013)$ & (3) \\
\hline 1996bo & $B V R I$ & 0.017 & 0.949 & $0.078(0.008)$ & $0.323(0.007)$ & (3) \\
\hline 1996C & $B V R I$ & 0.030 & 1.112 & $0.014(0.001)$ & $0.120(0.016)$ & (3) \\
\hline 1996X & $U B V R I$ & 0.007 & 0.890 & $0.069(0.007)$ & $0.011(0.006)$ & (3) \\
\hline $1996 Z$ & $B V R$ & 0.008 & 0.819 & $0.063(0.006)$ & $0.425(0.019)$ & (3) \\
\hline 1997dg & $U B V R I$ & 0.030 & 0.826 & $0.078(0.002)$ & $0.018(0.015)$ & (3) \\
\hline 1997do & $U B V R I$ & 0.010 & 0.937 & $0.063(0.002)$ & $0.073(0.010)$ & (3) \\
\hline 1997E & $U B V R I$ & 0.013 & 0.830 & $0.124(0.002)$ & $0.080(0.006)$ & (3) \\
\hline 1997Y & $U B V R I$ & 0.017 & 0.875 & $0.017(0.002)$ & $0.031(0.010)$ & (3) \\
\hline $1998 \mathrm{ab}$ & $U B V R I$ & 0.028 & 0.938 & $0.017(0.002)$ & $0.104(0.008)$ & (3) \\
\hline 1998bu & $U B V R I$ & 0.003 & 0.953 & $0.025(0.003)$ & $0.344(0.004)$ & (3) \\
\hline 1998dh & $U B V R I$ & 0.008 & 0.887 & $0.068(0.002)$ & $0.112(0.009)$ & (3) \\
\hline 1998dx & $U B V R I$ & 0.054 & 0.818 & $0.041(0.002)$ & $-0.048(0.018)$ & (3) \\
\hline $1998 \mathrm{ef}$ & $U B V R I$ & 0.018 & 0.871 & $0.073(0.002)$ & $-0.008(0.009)$ & (3) \\
\hline 1998eg & $U B V R I$ & 0.024 & 0.992 & $0.123(0.002)$ & $0.060(0.016)$ & (3) \\
\hline 1998es & $U B V R I$ & 0.010 & 1.074 & $0.032(0.002)$ & $0.143(0.008)$ & (3) \\
\hline $1998 \mathrm{~V}$ & $U B V R I$ & 0.017 & 0.930 & $0.196(0.002)$ & $0.033(0.007)$ & (3) \\
\hline 1999aa & $U B V R I$ & 0.014 & 1.068 & $0.040(0.002)$ & $0.003(0.005)$ & (3) \\
\hline 1999ac & $U B V R I$ & 0.010 & 1.111 & $0.046(0.002)$ & $0.091(0.006)$ & (3) \\
\hline $1999 \mathrm{cc}$ & $U B V R I$ & 0.031 & 0.822 & $0.023(0.002)$ & $0.019(0.017)$ & (3) \\
\hline 1999dk & $U B V R I$ & 0.015 & 1.042 & $0.054(0.002)$ & $0.155(0.008)$ & (3) \\
\hline 1999dq & $U B V R I$ & 0.014 & 1.062 & $0.110(0.002)$ & $0.160(0.005)$ & (3) \\
\hline 1999ef & $U B V R I$ & 0.039 & 1.033 & $0.087(0.002)$ & $-0.007(0.016)$ & (3) \\
\hline 1999ej & $U B V R I$ & 0.013 & 0.795 & $0.071(0.002)$ & $0.027(0.019)$ & (3) \\
\hline
\end{tabular}


Table 1. continued.

\begin{tabular}{|c|c|c|c|c|c|c|}
\hline SN & Band & $z$ & $s$ & $E(B-V)^{M W}$ & $E(B-V)^{H G}$ & Ref. \\
\hline 1999ek & $U B V R I$ & 0.018 & 0.914 & $0.561(0.002)$ & $0.179(0.006)$ & (3) \\
\hline 1999gd & $U B V R I$ & 0.019 & 0.957 & $0.041(0.002)$ & $0.486(0.013)$ & (3) \\
\hline 1999gp & $U B V R I$ & 0.027 & 1.212 & $0.056(0.002)$ & $0.126(0.003)$ & (3) \\
\hline 2000B & $U B V R I$ & 0.020 & 0.970 & $0.068(0.002)$ & $0.229(0.011)$ & (3) \\
\hline 2000ca & $U B V$ & 0.024 & 1.011 & $0.067(0.002)$ & $-0.040(0.007)$ & (4a) \\
\hline 2000ce & $U B V R I$ & 0.016 & 1.076 & $0.057(0.002)$ & $0.535(0.009)$ & (3) \\
\hline 2000cf & $U B V R I$ & 0.036 & 0.931 & $0.032(0.002)$ & $-0.001(0.014)$ & (3) \\
\hline 2000fa & $U B V R I$ & 0.022 & 0.992 & $0.069(0.002)$ & $0.091(0.008)$ & (3) \\
\hline 2001ba & $B V$ & 0.029 & 1.025 & $0.064(0.002)$ & $-0.021(0.009)$ & (4a) \\
\hline $2001 b t$ & $B V$ & 0.015 & 0.875 & $0.065(0.002)$ & $0.232(0.006)$ & (4b) \\
\hline $2001 c z$ & $U B V$ & 0.015 & 1.007 & $0.092(0.002)$ & $0.146(0.006)$ & $(4 b)$ \\
\hline 2001el & $U B V R I$ & 0.004 & 0.962 & $0.069(0.002)$ & $0.168(0.003)$ & (4) \\
\hline $2001 \mathrm{~V}$ & $B V R I$ & 0.015 & 1.120 & $0.020(0.002)$ & $0.128(0.019)$ & (4) \\
\hline 2002bo & $U B V R I$ & 0.004 & 0.900 & $0.025(0.002)$ & $0.436(0.006)$ & $(4 b, 5)$ \\
\hline 2002er & $U B V R I$ & 0.009 & 0.896 & $0.160(0.002)$ & $0.195(0.009)$ & (6) \\
\hline 2003du & $U B V R I$ & 0.006 & 0.991 & $0.010(0.002)$ & $-0.069(0.001)$ & (7) \\
\hline $2005 \mathrm{cf}$ & UBVRI & 0.006 & 0.958 & $0.097(0.002)$ & $0.028(0.002)$ & (8) \\
\hline
\end{tabular}
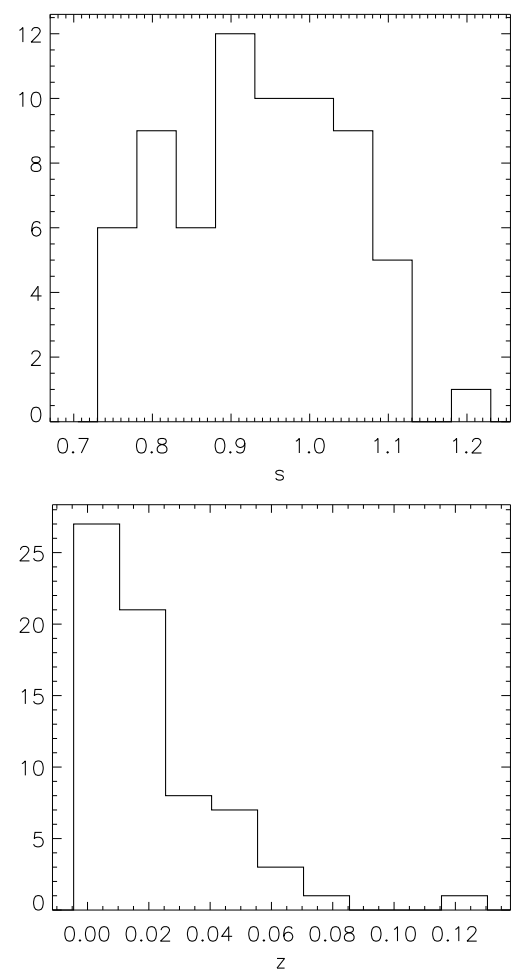

Fig. 1. Distribution of the $B$-band stretch factor, $s$, (top panel), distribution of the redshift (bottom panel) for the $69 \mathrm{SNe}$ that have experienced small amounts of extinction.

The spectral-template colours are modified using a cubic-spline interpolation of the ratio between the synthetic photometry and the new photometry at the effective wavelengths, for the $U, B, V$, $R$ and $I$-band. These are determined from the fitted colour curves as follows:

$$
\begin{aligned}
& U=(U-B)+B^{\prime} \\
& B=B^{\prime} \\
& V=B^{\prime}-(B-V) \\
& R=V-(V-R) \\
& I=R-(R-I)
\end{aligned}
$$

where $B^{\prime}$ indicates the $B$-band lightcurve by Goldhaber et al. (2001), and the colours given within brackets are the ones fitted in the previous iteration.
The steps 1-7 were repeated until the difference in the colour-curves between consecutive iterations changed by less than $0.5 \%$. Only three iterations were required to achieve this precision.

Tables 2-8 report the values for $a$ and $b$ for each colour, as a function of time. Figure 2 shows the time evolution of $U-B$, $B-V, V-R$, and $R-I$ for different values of the stretch factor, $s=0.8,0.9,1.0,1.1,1.2$. Figure 3 provides a comparison of the $a$ parameter fitted for each of the colours. For $U-B$, the dependence on stretch is significant even before maximum light, and decreases with time (see discussion in Sect. 8). In all other cases, the dependence on stretch peaks between 15 and 20 days after $B$-band maximum. This coincides with the epoch at which the photosphere recedes into the core of the supernova, and absorption features from iron-group elements appear in the spectrum. Kasen \& Woosley (2007) show that the colour evolution depends on the ionization evolution of the iron-group elements, which is more rapid for supernovae that are dimmer. Their model dependence is in good agreement with the results of our present work.

We note that the data points were assumed to be uncorrelated at different epochs when performing the fit. Thus, even if some SN lightcurve are better sampled than others, all points have similar weight in the fitting. Since this is only a crude approximation, we take this into account in the determination of the uncertainties. For this reason, the uncertainties in the colour curves reported in Tables 2-8, are determined using Monte Carlo simulations, as explained in Sect. 7.

\section{Intrinsic colour dispersion}

When applied to the data, the colour-stretch relation that we derive decreases the dispersion in the colour curve, for all colours. The residual dispersion in the colour curve is, however, too significant to be explained entirely by the statistical fluctuations of the measurement errors. This implies that the residual dispersion is due to an intrinsic variation in the SNe colours. We follow a procedure similar to that of Nobili et al. (2003) to estimate the intrinsic dispersion in each colour as a function of time. The data, corrected for the colour-stretch relation derived in the previous section, are divided into time bins. For each time bin, we compute the weighted average of the residual to the colour curve (for a $s=1$ supernova), and the sample-weighted standard 
Table 2. $U-B$ : time evolution of the $a$ and $b$ parameters. $c_{U-B}=0.82 \pm$ 0.06

\begin{tabular}{ccc}
\hline \hline Epoch & $b(t)$ & $a(t)$ \\
\hline-9 & $-0.614 \pm 0.011$ & $-1.046 \pm 0.101$ \\
-6 & $-0.594 \pm 0.011$ & $-1.002 \pm 0.090$ \\
-3 & $-0.563 \pm 0.010$ & $-0.940 \pm 0.077$ \\
0 & $-0.462 \pm 0.008$ & $-0.779 \pm 0.058$ \\
3 & $-0.325 \pm 0.009$ & $-0.635 \pm 0.078$ \\
6 & $-0.196 \pm 0.009$ & $-0.648 \pm 0.093$ \\
9 & $-0.102 \pm 0.008$ & $-0.848 \pm 0.097$ \\
12 & $-0.032 \pm 0.009$ & $-1.074 \pm 0.125$ \\
15 & $0.025 \pm 0.011$ & $-1.153 \pm 0.145$ \\
18 & $0.079 \pm 0.011$ & $-0.959 \pm 0.136$ \\
21 & $0.126 \pm 0.011$ & $-0.586 \pm 0.151$ \\
24 & $0.161 \pm 0.012$ & $-0.183 \pm 0.184$ \\
27 & $0.173 \pm 0.012$ & $0.107 \pm 0.174$ \\
30 & $0.165 \pm 0.011$ & $0.260 \pm 0.136$ \\
33 & $0.149 \pm 0.013$ & $0.311 \pm 0.124$ \\
36 & $0.136 \pm 0.014$ & $0.305 \pm 0.134$ \\
39 & $0.128 \pm 0.014$ & $0.274 \pm 0.128$ \\
42 & $0.123 \pm 0.012$ & $0.241 \pm 0.129$ \\
45 & $0.120 \pm 0.012$ & $0.224 \pm 0.135$ \\
48 & $0.118 \pm 0.014$ & $0.217 \pm 0.140$ \\
51 & $0.115 \pm 0.018$ & $0.209 \pm 0.145$ \\
54 & $0.113 \pm 0.023$ & $0.202 \pm 0.151$ \\
57 & $0.111 \pm 0.028$ & $0.194 \pm 0.158$ \\
60 & $0.109 \pm 0.033$ & $0.187 \pm 0.166$ \\
63 & $0.107 \pm 0.039$ & $0.180 \pm 0.174$ \\
\hline
\end{tabular}

deviation, as the square root of the weighted second moment (see Nobili et al. 2003). Table 3 gives the results of this analysis.

The weighted standard deviation can be taken as an estimate of the intrinsic dispersion in each time bin, $\Delta$. As pointed out by Nobili et al. (2003), however, this is an overestimate because part of the scatter is due only to measurement uncertainty. To disentangle both contributions, we execute a Monte Carlo simulation to generate synthetic data sets, which have dispersions in their measurements that correspond only to the quoted measurement uncertainties. Thus, we compute the weighed standard deviation on the simulated data sets, $\delta$. We then complete a hypothesis test, to compare the dispersion measured in the real data to those measured in the synthetic data sets, for each colour.

We set a level of significance $\alpha=0.01$ for rejecting the null hypothesis, i.e. the probability that $\Delta \neq \delta$, under the assumption that the null hypothesis is correct, is assumed to be equal to $1 \%$ (Nobili et al. 2003; Cowan 1998). In all cases for which the null hypothesis is rejected, we compute the quantity:

$\Delta^{\mathrm{corr}}=\sqrt{\Delta^{2}-\delta^{2}}$

that we assumed to be an estimate of the intrinsic dispersion, and a lower limit to this value is set at a $99 \%$ confidence limit. We found no cases compatible with no intrinsic dispersion (see Table 3).

\section{Supernova spectral template}

The composite spectral template created by Hsiao et al. (2007), "HSIAO" in our plots, was used as the input to the first iteration of our analysis method. The template was produced by combining 600 spectra of $100 \mathrm{SNe}$ Ia; each observed spectrum was first colour-corrected to match a single SED and all spectra were then average-combined into a single spectral template. The colours used were those calculated by Knop et al. (2003),
Table 3. Results of the analysis of all SNe. First column: central value in days for each time bin; $N_{k}$ is the number of points for each bin; $\Delta_{X Y}$ is the intrinsic dispersion computed as weighted standard deviation; $\Delta_{X Y}^{\text {corr }}$ is the corrected intrinsic dispersion, computed as in Eq. (3); and in the last column we provide the estimated lower limit at 99\% C.L. (see text).

\begin{tabular}{rrccc}
\hline \hline Day & $N_{k}$ & $\Delta_{U B}$ & $\Delta_{U B}^{\text {corr }}$ & L.L. \\
\hline-10 & 22 & $0.13 \pm 0.03$ & 0.13 & 0.09 \\
0 & 83 & $0.10 \pm 0.03$ & 0.10 & 0.06 \\
10 & 67 & $0.06 \pm 0.05$ & 0.06 & 0.03 \\
20 & 44 & $0.10 \pm 0.02$ & 0.09 & 0.06 \\
30 & 29 & $0.06 \pm 0.07$ & 0.06 & 0.04 \\
40 & 20 & $0.07 \pm 0.02$ & 0.06 & 0.05 \\
\hline Day & $N_{k}$ & $\Delta_{B V}$ & $\Delta_{B V}^{\text {corr }}$ & L.L. \\
-5 & 77 & $0.05 \pm 0.01$ & 0.05 & 0.03 \\
0 & 180 & $0.03 \pm 0.01$ & 0.03 & 0.02 \\
10 & 228 & $0.08 \pm 0.01$ & 0.08 & 0.04 \\
18 & 165 & $0.05 \pm 0.01$ & 0.05 & 0.02 \\
30 & 137 & $0.10 \pm 0.01$ & 0.09 & 0.05 \\
40 & 74 & $0.06 \pm 0.01$ & 0.06 & 0.03 \\
50 & 66 & $0.12 \pm 0.04$ & 0.12 & 0.06 \\
\hline Day & $N_{k}$ & $\Delta_{V R}$ & $\Delta_{V R}^{\text {corr }}$ & L.L. \\
-5 & 64 & $0.04 \pm 0.01$ & 0.04 & 0.02 \\
0 & 120 & $0.04 \pm 0.01$ & 0.04 & 0.02 \\
10 & 131 & $0.03 \pm 0.01$ & 0.03 & 0.02 \\
15 & 65 & $0.08 \pm 0.01$ & 0.08 & 0.04 \\
20 & 82 & $0.03 \pm 0.01$ & 0.03 & 0.01 \\
30 & 81 & $0.03 \pm 0.05$ & 0.03 & 0.02 \\
40 & 58 & $0.05 \pm 0.01$ & 0.05 & 0.03 \\
50 & 47 & $0.03 \pm 0.01$ & 0.03 & 0.02 \\
\hline Day & $N_{k}$ & $\Delta_{R I}$ & $\Delta_{R I}^{\text {corr }}$ & L.L. \\
-10 & 44 & $0.04 \pm 0.01$ & 0.04 & 0.03 \\
0 & 147 & $0.06 \pm 0.01$ & 0.06 & 0.03 \\
10 & 141 & $0.10 \pm 0.02$ & 0.10 & 0.05 \\
20 & 77 & $0.13 \pm 0.02$ & 0.13 & 0.07 \\
25 & 97 & $0.05 \pm 0.02$ & 0.05 & 0.03 \\
40 & 62 & $0.14 \pm 0.03$ & 0.14 & 0.08 \\
50 & 50 & $0.14 \pm 0.04$ & 0.14 & 0.08 \\
\hline & & & &
\end{tabular}

for a "normal" $s=1 \mathrm{SNe}$ Ia. The HSIAO spectral template is meant to be used for computing $K$-corrections after it has been corrected for the observed colours. This has the advantage of being independent of an assumed stretch-colour relation, but the resulting uncertainties in the $K$-corrections, depend on the availability of observed colours, and can be as large as $0.12 \mathrm{mag}$, as showed in Fig. 9 of Hsiao et al. (2007). Using the colourstretch relation derived with our technique, the spectral-template colours can be adjusted for each supernova lightcurve shape, before computing $K$-corrections. In our case, the uncertainties on $K$-corrections are dominated by the intrinsic dispersion in the supernova colours. Whether these correspond to an intrinsic variability of the spectral features is beyond the scope of this work. We note, however, that there are several indications that at least some of the characteristic SN Ia spectral features correlate well with lightcurve shape parameters (Nugent et al. 1995; Benetti et al. 2005; Garavini et al. 2007).

Another interesting approach is the one adopted by Guy et al. (2007) while developping the lightcurve fitter SALT2. Instead of building spectral templates to be used for computing $K$-corrections, an empirical model that describes the time variation of the spectral energy distributions and its dependence on a lightcurve-shape parameter (corresponding to stretch), is used. In this case, the SED is a function of epoch, wavelength, and lightcurve-shape parameter. This ambitious model is trained on 
Table 4. Comparison at different redshifts between the $K$-corrections computed using the various spectral templates for $s=1$. The corrections are computed from the band listed in the first column to rest-frame $B$-band. The mean and the rms are computed over time on the residuals to the $K$-corrections computed using our templates. The largest dispersion is noted at $z=0.6$ where the mismatch between observed and rest-frame filters is at its maximum, as also noted by Hsiao et al. (2007).

\begin{tabular}{crrr}
\hline \hline Band & $z$ & mean & rms \\
\hline HSIAO & & & \\
$B$ & 0.1 & 0.008 & 0.020 \\
$V$ & 0.3 & 0.007 & 0.015 \\
$R$ & 0.6 & 0.012 & 0.031 \\
$I$ & 0.8 & -0.011 & 0.028 \\
$I$ & 0.9 & 0.005 & 0.010 \\
\hline SALT2 & & & \\
$B$ & 0.1 & 0.023 & 0.017 \\
$V$ & 0.3 & 0.021 & 0.015 \\
$R$ & 0.6 & 0.041 & 0.040 \\
$I$ & 0.8 & -0.040 & 0.026 \\
$I$ & 0.9 & 0.016 & 0.013 \\
\hline
\end{tabular}

Table 5. Comparison between the dependence of $K$-corrections on stretch for two different redshifts, for SALT2 and our templates. The mean and the rms are computed over time on the residuals to our templates. Once again the largest dispersion is noted at $z=0.6$, where the mismatch between observed and rest-frame filters is maximum, and for more extreme stretch values.

\begin{tabular}{lllr}
\hline \hline$z$ & $s$ & mean & rms \\
\hline 0.3 & 0.8 & 0.014 & 0.045 \\
0.3 & 0.9 & 0.019 & 0.028 \\
0.3 & 1.0 & 0.022 & 0.015 \\
0.3 & 1.1 & 0.023 & 0.013 \\
0.6 & 0.8 & 0.061 & 0.123 \\
0.6 & 0.9 & 0.049 & 0.078 \\
0.6 & 1.0 & 0.042 & 0.041 \\
0.6 & 1.1 & 0.036 & 0.033 \\
\hline
\end{tabular}

a data set that overlaps largely with the one used to construct the HSIAO templates. Due to the larger number of parameters required to describe the model, the uncertainties are however quite large, especially in the $U$ and $I$ part of the spectrum, where fewer measurements are available.

A comparison between the spectral templates for a $s=1 \mathrm{su}-$ pernova, at different epochs from maximum light (day $=-10$, $0,10,20)$, is shown in Fig. 5. Differences between our colourcorrected spectral template and the original HSIAO template are small, up to $2 \%$, and even smaller around maximum light, about $0.5 \%$. This differences originate in the different data sets used to determine intrinsic colours. The HSIAO-template uses the colours measured by Knop et al. (2003), which are based on a smaller data set than that used in this study. We note, in particular, that the $U-B$ colour was derived using only measurements of $5 \mathrm{SNe}$ Ia at maximum. When comparing our spectral template, with the SALT 2 template we find large differences even for $s=1$. We note, however, that the differences are mainly for the part of the spectra that corresponds to the $U$-band, where large uncertainties in the SALT2 templates are reported by the authors.

To further compare the spectral templates, we computed $K$-corrections using SALT2 templates, HSIAO templates, and our own templates for different values of the redshift. The results of the comparison for $s=1$ are reported in Table 4 . The larger
Table 6. $B-V$ : time evolution of the $a$ and $b$ parameters. $c_{B-V}=1$ by construction.

\begin{tabular}{ccc}
\hline \hline Epoch & $b(t)$ & $a(t)$ \\
\hline-9 & $-0.116 \pm 0.006$ & $-0.207 \pm 0.050$ \\
-6 & $-0.104 \pm 0.005$ & $-0.187 \pm 0.045$ \\
-3 & $-0.092 \pm 0.004$ & $-0.167 \pm 0.040$ \\
0 & $-0.067 \pm 0.004$ & $-0.135 \pm 0.033$ \\
3 & $0.001 \pm 0.004$ & $-0.116 \pm 0.034$ \\
6 & $0.089 \pm 0.005$ & $-0.267 \pm 0.041$ \\
9 & $0.186 \pm 0.004$ & $-0.626 \pm 0.040$ \\
12 & $0.306 \pm 0.005$ & $-1.033 \pm 0.052$ \\
15 & $0.462 \pm 0.006$ & $-1.316 \pm 0.058$ \\
18 & $0.652 \pm 0.005$ & $-1.380 \pm 0.051$ \\
21 & $0.838 \pm 0.005$ & $-1.272 \pm 0.056$ \\
24 & $0.986 \pm 0.006$ & $-1.038 \pm 0.064$ \\
27 & $1.067 \pm 0.006$ & $-0.727 \pm 0.054$ \\
30 & $1.097 \pm 0.006$ & $-0.398 \pm 0.046$ \\
33 & $1.092 \pm 0.008$ & $-0.109 \pm 0.059$ \\
36 & $1.071 \pm 0.008$ & $0.082 \pm 0.066$ \\
39 & $1.044 \pm 0.007$ & $0.156 \pm 0.052$ \\
42 & $1.014 \pm 0.008$ & $0.158 \pm 0.030$ \\
45 & $0.980 \pm 0.008$ & $0.134 \pm 0.016$ \\
48 & $0.944 \pm 0.007$ & $0.122 \pm 0.014$ \\
51 & $0.906 \pm 0.007$ & $0.123 \pm 0.014$ \\
54 & $0.868 \pm 0.009$ & $0.128 \pm 0.019$ \\
57 & $0.830 \pm 0.013$ & $0.129 \pm 0.021$ \\
60 & $0.791 \pm 0.018$ & $0.131 \pm 0.023$ \\
63 & $0.752 \pm 0.023$ & $0.133 \pm 0.025$ \\
\hline & &
\end{tabular}

discrepancy is found for $z=0.6$, where the mismatch between the observed and rest-frame filter is at its largest. This agrees with the results of Hsiao et al. (2007), which suggests that there is an uncertainty of $\sim 0.04$ in the $K$-corrections at this redshift.

A comparison between the dependence of $K$-corrections on stretch, is only possible between SALT 2 and our templates. Figure 6 shows the $K$-corrections to rest-frame $B$-band for two different redshifts of $z=0.3$ and $z=0.6$, that were computed using the two sets of templates. The rms in the difference is reported in Table 5.

All differences measured indicate that systematic uncertainties as large as 0.04 mag can be introduced using the uncorrected spectral template, which limits our ability to use SNe Ia for precision cosmology.

\section{The reddening law derived from $\mathrm{SNe}$ la}

Corrections due to the wavelength dependence of the dimming of the supernova light is one of the largest systematic uncertainties in supernova cosmology. While empirical evidence for the dependence of peak magnitudes on SN colours is very clear, there is no consensus on how to distinguish between the contributions of reddening due to dust in the host galaxy and intrinsic SN colour variations. Most published results assume that the dust in the host galaxy to have similar properties as (the average) Milky Way dust, which implies a total-to-selective extinction parameter of $R_{V}=3.1$. Studies of the dust properties of a small number of distant galaxies indicate mean values that are compatible with this value, even though a significant range of values are measured (Ostman et al. 2007; Goudfrooij et al. 1994; Patil et al. 2007). Ideally, cosmological distance estimations that use SNe Ia should include an extinction correction for each line of sight, which includes the specific dust properties of each host galaxy. As shown in Nordin et al. (2008), however, the 
Table 7. $V-R$ : time evolution of the $a$ and $b$ parameters. $c_{V-R}=0.37 \pm$ 0.03 .

\begin{tabular}{ccc}
\hline \hline Epoch & $b(t)$ & $a(t)$ \\
\hline-9 & $0.010 \pm 0.007$ & $-0.543 \pm 0.049$ \\
-6 & $0.008 \pm 0.006$ & $-0.513 \pm 0.044$ \\
-3 & $0.006 \pm 0.005$ & $-0.484 \pm 0.039$ \\
0 & $0.000 \pm 0.005$ & $-0.418 \pm 0.036$ \\
3 & $-0.020 \pm 0.007$ & $-0.247 \pm 0.050$ \\
6 & $-0.058 \pm 0.007$ & $-0.064 \pm 0.054$ \\
9 & $-0.101 \pm 0.006$ & $0.018 \pm 0.059$ \\
12 & $-0.118 \pm 0.007$ & $-0.127 \pm 0.077$ \\
15 & $-0.082 \pm 0.006$ & $-0.565 \pm 0.061$ \\
18 & $-0.001 \pm 0.008$ & $-0.967 \pm 0.072$ \\
21 & $0.101 \pm 0.007$ & $-1.016 \pm 0.064$ \\
24 & $0.197 \pm 0.008$ & $-0.828 \pm 0.065$ \\
27 & $0.262 \pm 0.008$ & $-0.574 \pm 0.066$ \\
30 & $0.294 \pm 0.007$ & $-0.336 \pm 0.063$ \\
33 & $0.300 \pm 0.008$ & $-0.138 \pm 0.078$ \\
36 & $0.285 \pm 0.009$ & $0.002 \pm 0.084$ \\
39 & $0.257 \pm 0.007$ & $0.075 \pm 0.073$ \\
42 & $0.228 \pm 0.008$ & $0.097 \pm 0.080$ \\
45 & $0.205 \pm 0.010$ & $0.086 \pm 0.091$ \\
48 & $0.190 \pm 0.009$ & $0.057 \pm 0.083$ \\
51 & $0.180 \pm 0.008$ & $0.025 \pm 0.083$ \\
54 & $0.172 \pm 0.009$ & $0.005 \pm 0.098$ \\
57 & $0.167 \pm 0.012$ & $-0.000 \pm 0.104$ \\
60 & $0.162 \pm 0.017$ & $-0.005 \pm 0.109$ \\
63 & $0.158 \pm 0.023$ & $-0.010 \pm 0.116$ \\
\hline & &
\end{tabular}

Table 8. $R-I$ : time evolution of the $a$ and $b$ parameters. $c_{R-I}=0.40 \pm$ 0.03 .

\begin{tabular}{ccc}
\hline \hline Epoch & $b(t)$ & $a(t)$ \\
\hline-9 & $-0.145 \pm 0.007$ & $-0.099 \pm 0.057$ \\
-6 & $-0.167 \pm 0.006$ & $-0.104 \pm 0.051$ \\
-3 & $-0.199 \pm 0.005$ & $-0.112 \pm 0.043$ \\
0 & $-0.296 \pm 0.005$ & $-0.151 \pm 0.035$ \\
3 & $-0.406 \pm 0.006$ & $-0.246 \pm 0.050$ \\
6 & $-0.467 \pm 0.007$ & $-0.412 \pm 0.057$ \\
9 & $-0.448 \pm 0.006$ & $-0.641 \pm 0.056$ \\
12 & $-0.366 \pm 0.007$ & $-0.869 \pm 0.072$ \\
15 & $-0.247 \pm 0.008$ & $-1.032 \pm 0.078$ \\
18 & $-0.112 \pm 0.007$ & $-1.079 \pm 0.061$ \\
21 & $0.019 \pm 0.008$ & $-1.029 \pm 0.064$ \\
24 & $0.128 \pm 0.008$ & $-0.908 \pm 0.072$ \\
27 & $0.210 \pm 0.007$ & $-0.742 \pm 0.065$ \\
30 & $0.266 \pm 0.008$ & $-0.549 \pm 0.071$ \\
33 & $0.291 \pm 0.009$ & $-0.348 \pm 0.083$ \\
36 & $0.286 \pm 0.008$ & $-0.157 \pm 0.081$ \\
39 & $0.255 \pm 0.008$ & $0.004 \pm 0.080$ \\
42 & $0.215 \pm 0.009$ & $0.118 \pm 0.089$ \\
45 & $0.175 \pm 0.010$ & $0.164 \pm 0.090$ \\
48 & $0.139 \pm 0.009$ & $0.139 \pm 0.085$ \\
51 & $0.106 \pm 0.008$ & $0.077 \pm 0.109$ \\
54 & $0.075 \pm 0.010$ & $0.030 \pm 0.139$ \\
57 & $0.046 \pm 0.013$ & $0.014 \pm 0.149$ \\
60 & $0.017 \pm 0.018$ & $-0.001 \pm 0.159$ \\
63 & $-0.013 \pm 0.023$ & $-0.017 \pm 0.170$ \\
\hline
\end{tabular}

variations in $R_{V}$ between lines of sight are much less problematic for cosmological applications than a potential bias in the adopted mean value.

Intriguingly, there are a large number of measurements of the mean $R_{V}$ obtained from $\mathrm{SNe}$ Ia, based on different methods, that disagree with the universally-assumed value: significantely smaller values of mean $R_{V}$ are found. Some examples are
$R_{V} \sim 1.8$ (Krisciunas et al. 2000), $R_{V}=2.55 \pm 0.30$ (Riess et al. 1996), $R_{V}=2.6 \pm 0.4$ (Phillips et al. 1999), $R_{V}=1.09$ (Tripp 1998), $R_{V}=2.5$ (Altavilla et al. 2004), and $\beta=1.77 \pm 0.16$ that corresponds to $R_{V}=0.77 \pm 0.16$ (Astier et al. 2006; Guy et al. 2007).

Using the fitted $c$ parameters in Eq. (1), the reddening law that provides the most appropriate description of the data can be determined. In fitting the $c$ parameter for each colour, $c_{U B}, c_{B V}, c_{V R}, c_{R I}$, we minimize the part of the colour dispersion that depends on reddening, without assuming any value for $R_{V}$. The four $c$ parameters, one for each colour fitted, can then be used to estimate the parameter $R_{V}$ :

$$
\begin{aligned}
c_{U B} & =R_{U}-R_{B} \\
c_{B V} & =R_{B}-R_{V} \\
c_{V R} & =R_{V}-R_{R} \\
c_{R I} & =R_{R}-R_{I}
\end{aligned}
$$

where $R_{X} \equiv A_{X} / E(B-V)$, and $A_{X}$ is the dust absorption in a given band. We note that $c_{B V}=1$ by construction (see Eq. (1)); we therefore have 3 independent equations. Figure 8 shows $A_{\lambda}-A_{B}$ as a function of wavelength for $E(B-V)=0.1$. The data points are determined using the $c$ parameters. The dotted line is the Cardelli, Claython \& Mathis (CCM) law assuming $R_{V}=3.1$ and the light grey solid line is the result of fitting the CCM law to our data points. The best fit is obtained for $R_{V}=1.01 \pm 0.25$. The dashed line is the result of a similar procedure obtained by (Guy et al. 2007) while developing the SALT2 templates. A typical value of $E(B-V)=0.1$ was used in analogy with the work of Guy et al. (2007). We note that our measurements agree well with the results from SALT 2 in the $R$ and $I$-bands, but disagree in the $U$-band. We note, that the SALT 2 curve is not easily modelled by simply assuming a different value for $R_{V}$ in CCM.

To test the effect of selecting a low-extincted sample, we introduced to the analysis 11 high-extincted SNe. We assumed the colour curves obtained for the low-extincted sample, and investigated the reddening law using the larger sample. The $c$ parameters derived in this way are slightly different, and correspond to a larger value of $R_{V}$ when fitted by the CCM law, equal to $R_{V}=1.75 \pm 0.27$. The two values found differ by about 3 standard deviations. Given that the two samples are not independent of each other, since $69 \mathrm{SNe}$ are present in both, this difference is even more important, and is worth additional investigations.

\section{Monte Carlo simulations}

Due to the potential correlation of the fitted parameters $a, b$, and $c$, and the possible correlation in the intrinsic dispersion, we used Monte Carlo simulations to estimate the uncertainties in the above parameters, as well as in $R_{V}$.

The data suggests that the intrinsic colour dispersion is correlated between points at different epochs, e.g. if a $\mathrm{SN}$ is bluer than average at a given epoch, it is likely to remain bluer ${ }^{5}$.

However, the correlation coefficient as a function of epoch is quite difficult to quantify, given the small sample and the sizable measurement uncertainties. Moreover, the inhomogeneity of the data sample could partially introduce such correlations, especially in the $U$-band, for which a false correlation could originate from problems in the $S$-corrections between filter systems (see

\footnotetext{
5 We note that, the colour excess $E(B-V)$ determined from the $B-V$ colour, is used in the fit of Eq. (1) for all of the other colours. Thus, a SN can still remain redder or bluer than average even after the reddening is taken into account. The reddening law that minimizes the scatter around all colours is the result of the method used.
} 

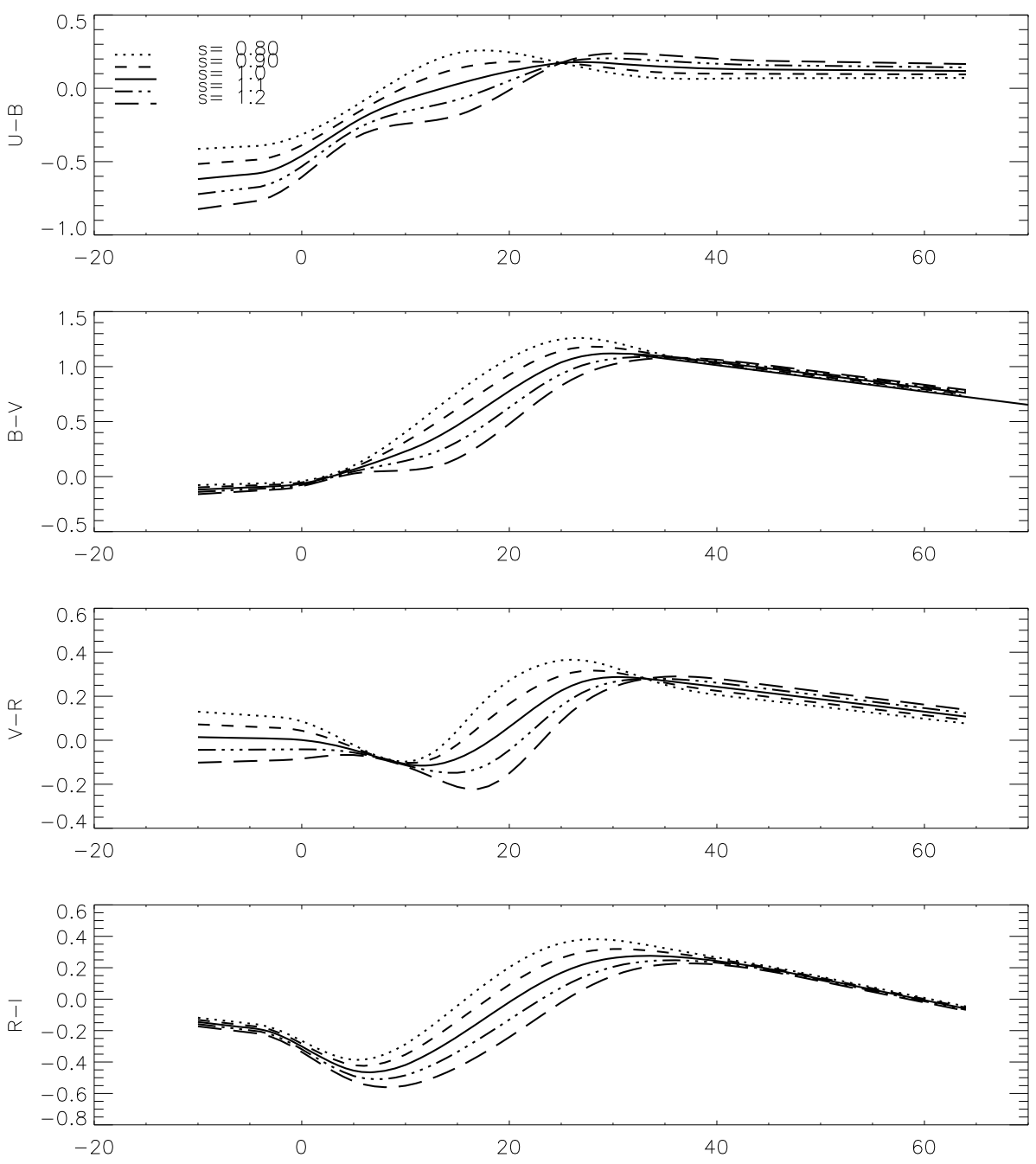

Fig. 2. From top to bottom, $U-B, B-V, V-R$, and $R-I$ time evolution for different stretch values: $s=0.8,0.9,1.0,1.1,1.2$. The straight line at late times in the $B-V$ evolution is the Lira line. The abscissa is the rest-frame epoch since the $B$-band maximum light.

also the discussion in the next section). For these reasons, we considered, when completing the Monte Carlo simulations, data uncertainty for the two extreme cases of either a full correlation or no correlation at all in the intrinsic dispersion (see Sect. 4). We found that the case of no correlation at all corresponds to the larger uncertainties in the fitted parameters, and we have conservatively chose these as our estimate of the uncertainties.

We generated 100 synthetic data samples, with as many lightcurves as in the real set, according to the following prescription:

- data points were taken from a normal distribution centred on the light curves; this distribution had a dispersion, that was given by the measurement uncertainties, which were all assumed to be uncorrelated, plus an intrinsic dispersion that was fully-correlated in time, i.e. all points for a given $\mathrm{SN}$, in a photometric band, had the same intrinsic dispersions at all epochs (case $A$ ), or was fully uncorrelated (case $B$ ). The intrinsic dispersion was considered to be described by a Gaussian function, with a standard deviation of $0.05 / \sqrt{2}^{6}$.

6 The value of 0.05 has been chosen a posteriori as average intrinsic dispersion in all colours at all epochs, given the results shown in Table 3. Using a smaller value, such as 0.03 , closer to the lower limit values in Table 3, we find smaller uncertainties on the parameters. In addition, we assumed the intrinsic dispersion in colours to be equally distributed in the two bands.
The data points were generated at the same epochs as the real data.

- We compute the colours and use the measured $E(B-V)$ to add reddening in each colour, given an assumed value of $R_{V}$.

- We fit the colour curves for the simulated samples, and retrieve the value of $R_{V}$ using the fitted $c$ parameters.

The dispersion in the fitted parameters, in all of the simulated data sets, was taken to be the uncertainty in the parameters, $a, b$, and $c$ that were fitted on the real data, as reported in Tables 2-8.

We run this test for two values: $R_{V}^{\text {(true) }}=1$ and $R_{V}^{\text {(true) }}=1.75$. In both cases, we were able to retrieve the true value, i.e. we found no bias in our method. The dispersion determined as rms of the distribution of the $R_{V}$, can be taken as an estimated upper limit to the uncertainties in the determination of $R_{V}$. We found a value of $\sigma=0.13$ (case $A$ ) and $\sigma=0.27$ (case $B$ ).

\section{Discussion}

We have presented an extended analysis on supernova colourcurves and their dependence on the light-curve shape parameter. An empirical model was defined, described by Eq. (1), and it was successfully fitted to the data. The robustness of the analysis, was thoroughly tested. The residuals from the colour curves were analysed to search for a further dependence on the stretch factor, SN redshift, or colour excess $E(B-V)$. No significant correlations were, however, found. The colour curves that we 

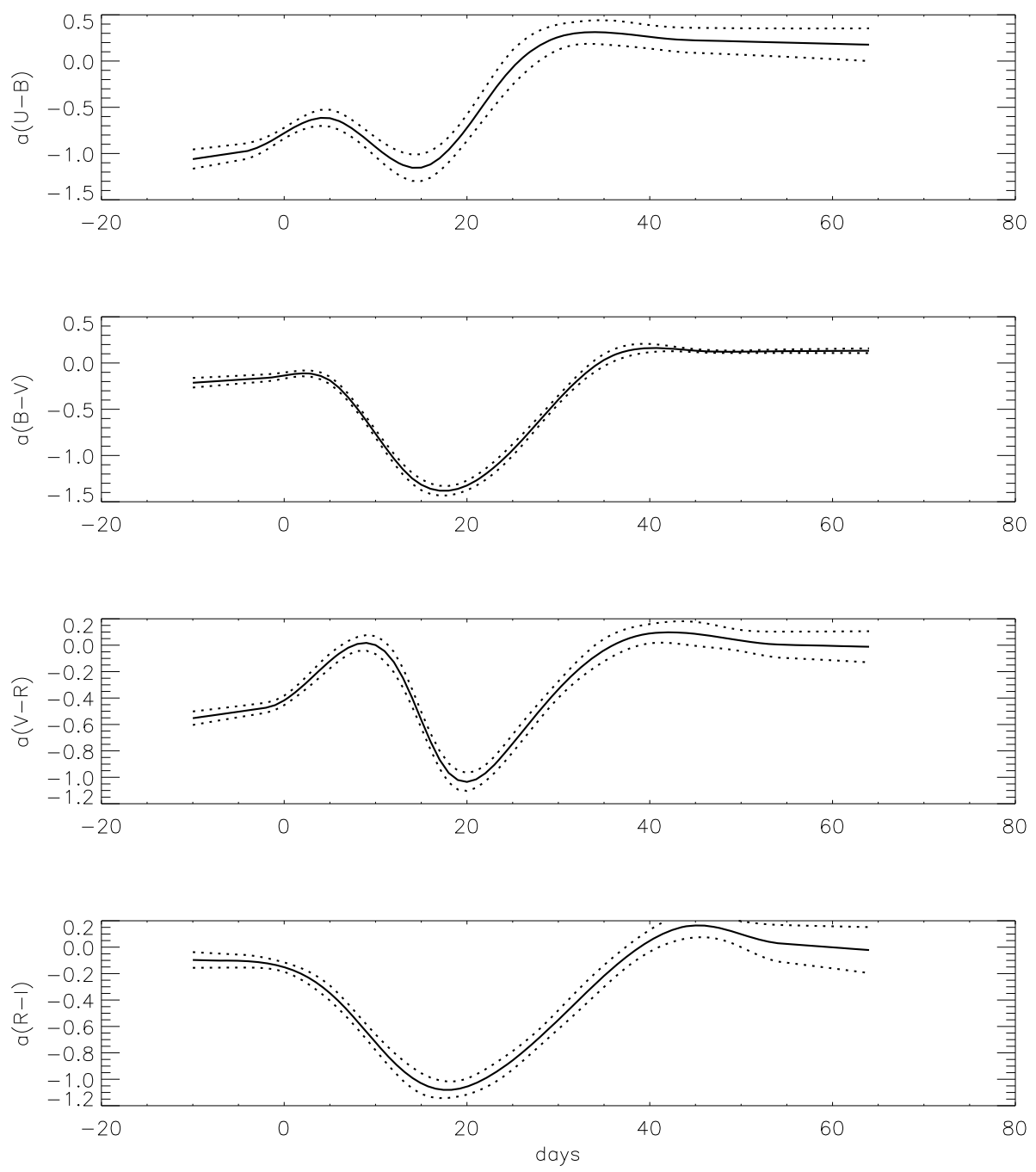

Fig. 3. From top to bottom, the $a$ parameter time evolution for $U-B, B-V, V-R$ and $R-I$ (solid line), and its uncertainty (dotted line). The minimum in the curves from top to bottom occurs at day $15,17,20$ and 20 .

derived, describe the subsample of very low-extinction objects, $E(B-V) \lesssim 0.05$, well, suggesting that the analysis was robust and not affected by moderately reddened SNe.

In general, the dispersion that we measured in all colours at all epochs was non-negligible, indicating that the colours of type Ia supernovae have more scatter than can be accounted for by the measurement uncertainties. These findings do not support earlier results that were based on a smaller sample, for which a negligible intrinsic scatter at late epochs was found in some colours (Nobili et al. 2003). The same conclusion was reached by Jha et al. (2007), when studying an overlapping sample of nearby $\mathrm{SNe}$ Ia. They found an intrinsic dispersion of $\sigma_{B-V}=0.062$ at epoch +35 , in agreement with the result reported here. The intrinsic dispersion for various epochs and colours is reported in Table 3 and could potentially be used to constrain and discriminate between different models of the physics of type Ia SNe. We notice, in general, a larger dispersion in the $U-B$ colour at all epochs than in all other colours. Due to the small amount of data available, it is difficult to establish whether the larger dispersion is an intrinsic characteristic of the $U$-band data or a consequence of systematic effects. The $U$-band photometry is affected, in general, by larger measurement uncertainties, smaller instrumental sensitivity, and larger extinction corrections (both atmospheric extinction, and interstellar extinction in the Milky Way and in the host galaxy). The large variability in the effective $U$-band bandpass shapes at different telescopes could increase the dispersion if accurate $S$-corrections are not applied (see e.g. the Appendix of Stanishev et al. 2007).The uncertainties possibly introduced by the colour term in the determination of the zero point, may be even larger. This is expected to be approximately $0.1 \mathrm{mag}$ in the $U$-band photometry (Suntzeff 2000). Many of these systematic uncertainties are correlated at different epochs, and thus, can easily simulate a correlated intrinsic dispersion similar to that observed in the data. This is certainly a strong concern for $U$-band, but it could be a second order effect for other bands as well. A larger and more homogeneous data set, such as that collected by the SDSS collaboration, would be a major improvement for investigating these hypotheses.

We compiled colour curves for HSIAO and SALT2 spectral templates by computing synthetic photometry, and compared them with our results. The left-hand panel of Fig. 9 shows a comparison of the colour curves, $U-B, B-V, V-R$, and $R-I$, for an $s=1$ supernova, as derived from the three spectral templates considered. The right-hand panel shows the colour curves for different stretch values for SALT2. We note that there is, in general, good agreement in $B-V$ colour curves for a standard $s=1$ supernova, with differences within the intrinsic colour dispersion. This is expected, since the availability of both $B$ and $V$ band data is excellent. The larger deviations between models are present in the $U-B$ colour curve. We note, in particular, the strong dependence on stretch shown by SALT 2 around day +40 . This is unexpected and most probably an artifact of a poorlyconstrained model, caused by too sparse data in the $U$-band at these late epochs. A similar reasoning holds for the behaviour at 

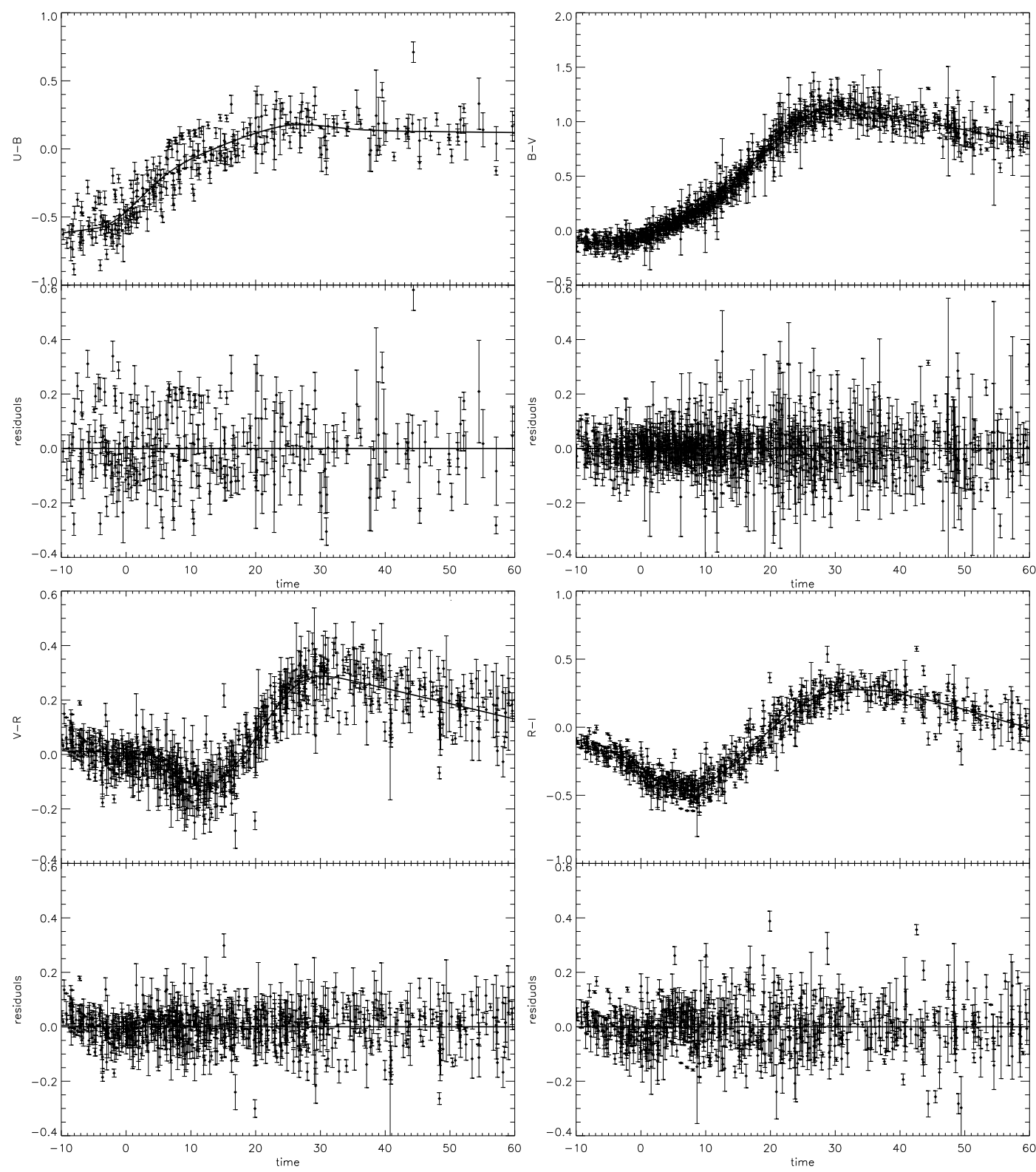

Fig. 4. From top to bottom, left to right, $U-B, B-V, V-R$ and $R-I$ time evolution compared with the data corrected for Milky Way extinction as well as for the colour-stretch relation and host galaxy extinction following Eq. (1).

early epochs. The SALT 2 colour curves show, overall, more wiggles than the colour curves derived in this work (see Fig. 2). One possible cause is the inhomogeneity in data quality. Some of the supernovae data have very small measurement errors and if no intrinsic colour dispersion is considered, they weight very heavily in the overall fit, possibly biasing the model. The comparison between spectral templates shown in Fig. 5 leads to similar conclusions. The SALT2 templates show a behaviour below $4000 \AA$ that is hard to attribute to real spectral features, both for early and late time (epoch $=-10$ and epoch $=+20$ ).

These fluctuations are not present in the HSIAO templates. Another anomaly in the SALT2 templates is the emission feature at $\sim 7600 \AA$, shown in many of the spectra after maximum. We note, however, that these anomalies are within the reported uncertainties in the SALT2 model.

When comparing with other published results, one should keep in mind the different aims of the various analyses. The SALT2 templates, for instance, are a side product of a light-curve fitter. The colour curves produced using synthetic photometry are a consequence of adjusting the spectra by the colours of the supernovae, and are not compiled for actual colour data. In this sense, they are subject to uncertainties introduced by the technique used for warping the spectra. Similarly, the HSIAO templates are contructed with the only aim of computing $K$-corrections, and not for fitting lightcurves or exploring colour properties. As mentioned in Sect. 5, the average colour of this template is that of Knop et al. (2003), which was based on the $U-B$ colour of the measurements of five supernovae at maximum. Another important difference is the much larger number of parameters used to compile SALT2 spectral templates, which caused larger uncertainties than this work and the HSIAO templates. When using a few parameters, one assumes implicitly some "resonable" constraints on the models, such as smoothness and the number of knots in the spline interpolation. The ability to relax these assumptions is limited by the quality and amount of available data. 

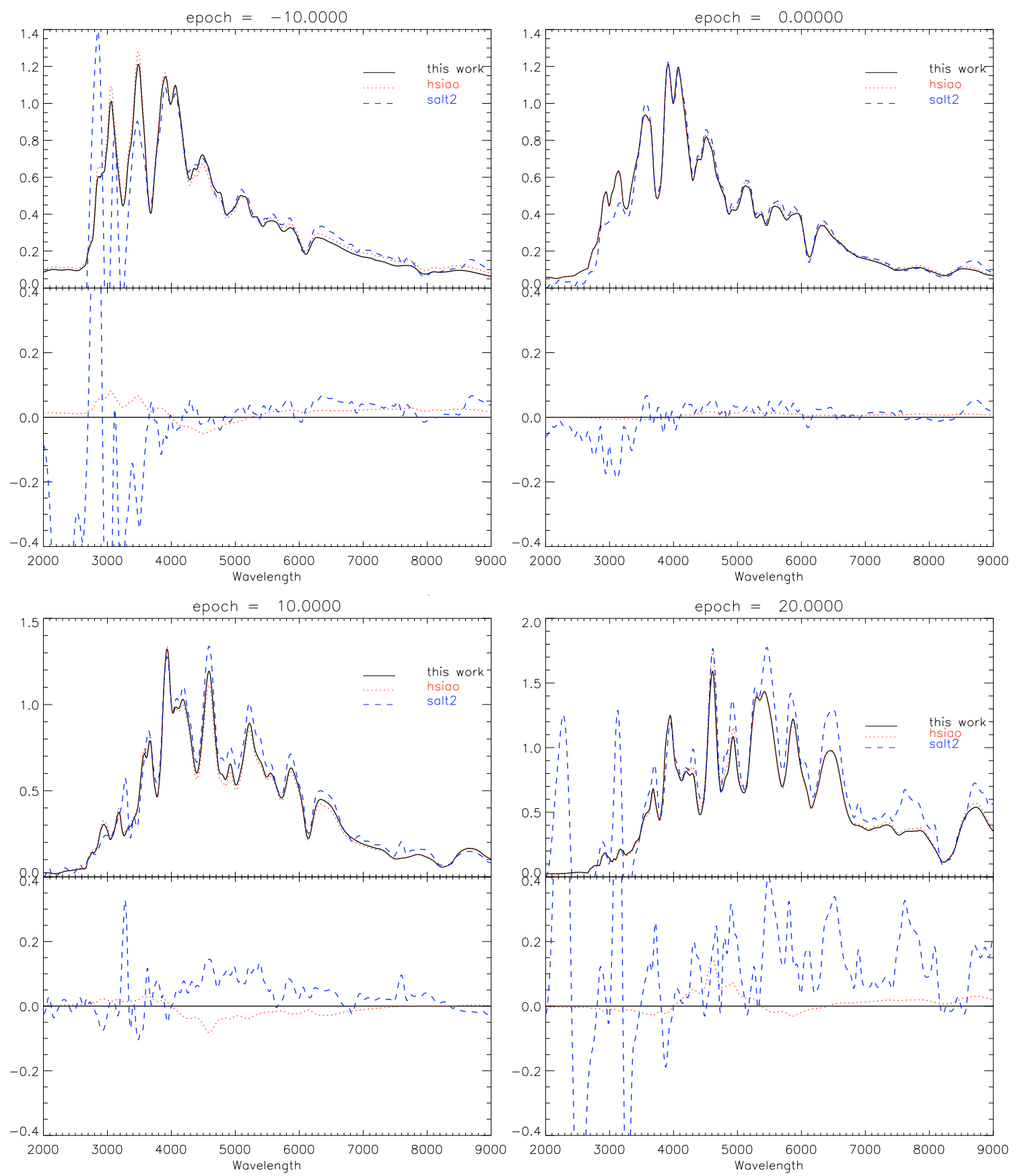

Fig. 5. Comparison of the spectral templates derived in this work for $s=1$ (black solid line), the spectral template by Guy et al. (2007) (blue dashed line) and the one by Hsiao et al. (2007) (red dashed-dotted line) for epochs $=-10,0,10,20$ referred to the time of maximum.

In Sect. 6, we derive a reddening law of our sample that is well described by the CCM law with a low value of $R_{V}=$ $1.01 \pm 0.25$ for SNe with $E(B-V)<0.25$, and $R_{V}=1.75$ for SNe with $E(B-V)<0.7$ mag. It should be noted that, we did not use the CCM law in any part of the analysis, apart from correcting the data for galactic reddening. The spectral templates are altered to match the average colour using a spline interpolation, and not the CCM curve. We assumed no prior on any value of $R_{V}$ or on any behaviour of the colour excess $E(B-V)$. Nevertheless, we find a good match to the CCM law. A direct comparison with the result derived by SALT2, indicates a very good agreement, apart from the $U$-band (see Fig. 8), with differences of about $0.06 \mathrm{mag}$, for $E(B-V)=0.1$. Given the intrinsic dispersion in $U-B$ colours, reported in Table 3 , this is not a large discrepancy.
Besides all concerns raised already about possible problems in the $U$-band data of the SALT2 templates, one should also considere the additional uncertainties that affects only the $U$-band, discussed above.

When adding the 11 highly-reddened $\mathrm{SNe}$ to our sample, up to $E(B-V)<0.7 \mathrm{mag}^{7}$, the derived extinction properties change, yielding a larger value of the total-to-selective extinction parameter $R_{V}=1.75 \pm 0.27$. The two values are statistically incompatible, given that they are determined using largely

7 We note that a few SNe Ia that show even higher reddening have recently been observed (Elias-Rosa et al. 2006, 2008). The values of $R_{V}$ determined for these individual $\mathrm{SNe}$ are in agreement with the larger value obtained in our work. 

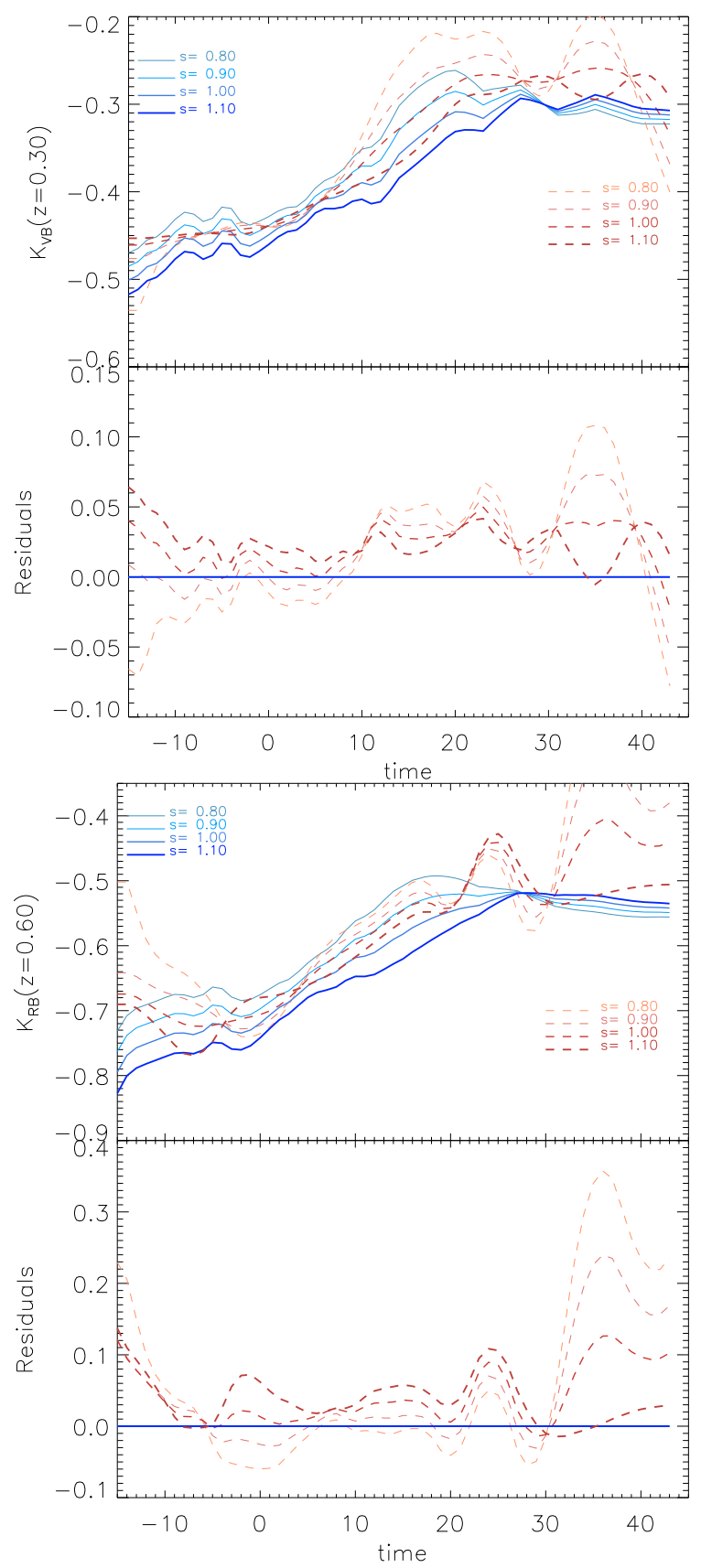

Fig. 6. $K$-corrections to restframe $B$-band at redshift $z=0.3$ (top panel) and $z=0.6$ (bottom panel) using our templates (solid lines) and SALT2 templates (dashed lines).

overlapping samples. As pointed out in Sect. 7, the quoted uncertainty may also be overestimated, since it was determined with the assumption of uncorrelated intrinsic dispersion between SN epochs. A lower uncertainty is found for the fully correlated case, $\sigma_{R_{V}} \sim 0.13$, which would make the difference even more significant.

Using highly-extincted $\mathrm{SNe}$ provides more leverage when studying extinction-law properties. Since we are unable to perform this analysis only on the small highly-extincted SN sample available, the determination of $R_{V}$ for the larger sample remains our most robust global estimate of the total-to-selective extinction parameter.

Based on our findings, we argue that it may be surprising that different analyses, based on overall minimization techniques

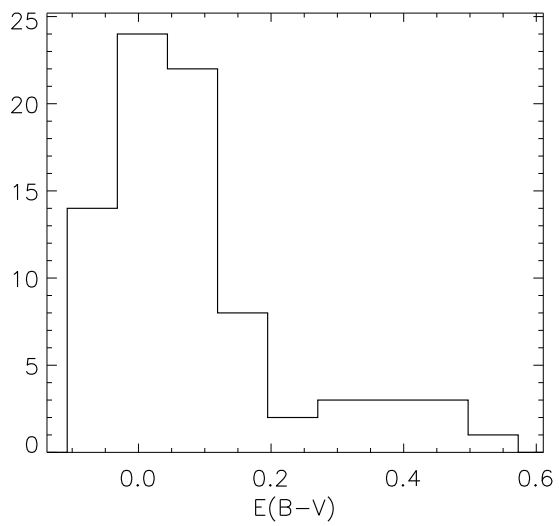

Fig. 7. Distribution of colour excess $E(B-V)$ for the entire sample including $80 \mathrm{SNe}$.

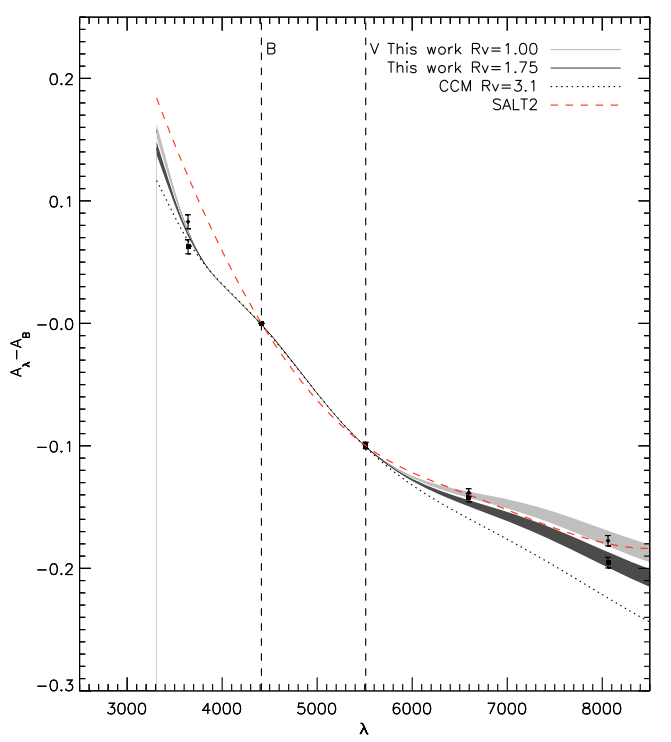

Fig. 8. The extinction law determined using the $c$ parameters in our model, compared with the one from Cardelli et al. (1989) with $R_{V}=$ 1.01 (light grey solid line) and $R_{V}=3.1$ (dotted line). Also plotted is the result for SALT2 (dashed red line). The CCM law with $R_{V}=1.01$ provides the best fit to our data (solid points). The lighted-shaded region represents the variability on the $R_{V}$ parameter, obtained by completing the Monte Carlo simulations. The darker-shaded region is obtained by adding 11 highly-extincted $\mathrm{SNe}$, and the CCM law with $R_{V}=1.75$ provides the best fit to our data (solid squares). A value of $E(B-V)=0.1$ was assumed for all the curves in the plot.

of the Hubble diagram, report different global fits of $R_{V}$, since its determination depends strongly both on the selection of the sample and the way in which the uncertainties are estimated. It is interesting to note that Tripp \& Branch (1999) found a very similar result when minimizing the dispersion in the Hubble diagram: $R_{V}=0.876$, which we infer from the measured $R_{B}=$ 1.876, when considering SNe with $E(B-V)<0.20 \mathrm{mag}$, and $R_{V}=1.439$, which we infer from the measured $R_{B}=2.439$, when this condition was relaxed.

Since most samples used to fit cosmological parameters are selected based on low reddening, caution should be taken in choosing the value of $R_{V}$ for reddening corrections. Even though the corrections could be small, a systematic bias may be introduced, which could lead to substantially different results, e.g. as shown by Conley et al. (2007).

The many studies reporting measurements of $R_{V}$ that are inconsistent with the Milky-Way value $\left(R_{V}=3.1\right)$ underline the 

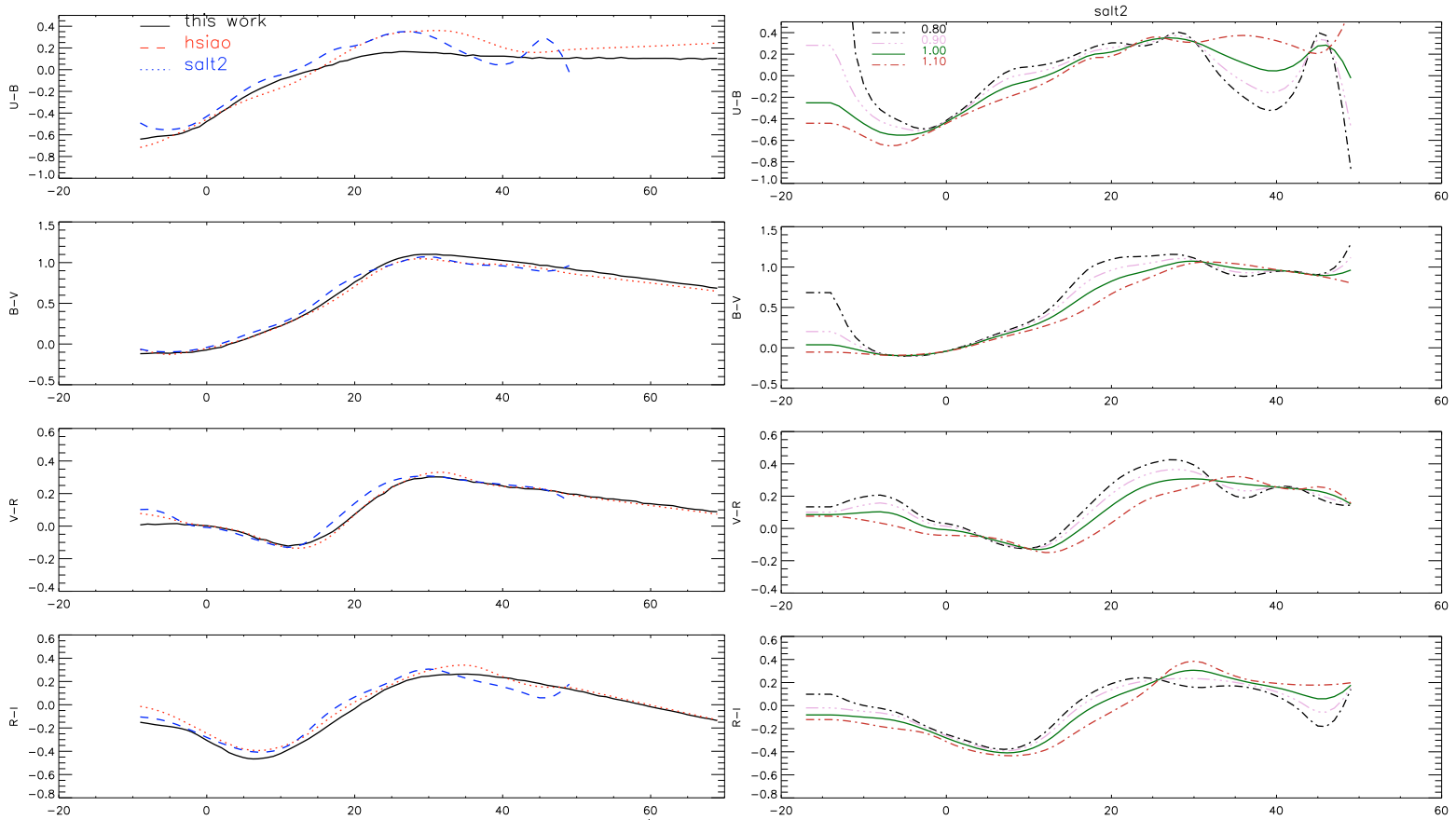

Fig. 9. Comparison of synthetic colour curves for all templates for $s=1$ supernova (left panel). Synthetic colour-curve evolution for different values of the stretch factor for the SALT2 templates (right panel).

need for a more rubust understanding of dust properties in other galaxies. In this work we have found that the reddening law for the low-extinction sample is statistically incompatible with that derived with the full available data set. This may be taken as evidence of additional processes being involved in producing the effective reddening law of SNe Ia, besides dimming by interstellar dust, e.g. scattered-light echoes due to dust in the circumstellar environment (Wang 2005; Patat et al. 2006; Wang et al. 2007), or intrinsic properties of the SN explosion, which mimick reddening by dust. Unfortunatelly, only a few high-quality colour measurements are currently available for highly-reddened $\mathrm{SNe}$ Ia. Additional observations of SNe Ia with $E(B-V)>0.2$ should be carried out to further investigate this issue.

Acknowledgements. The authors would like to thank the Göran Gustafsson Foundation and the Swedish Research Council for financial support. We are very grateful to Rick Kessler for pointing out the possibility of a bias in the determination of $R_{V}$. We would also like to thank Julien Guy for helpful discussions about the SALT2 templates. Vallery Stanishev is acknowledged for stimulating discussions. We acknowledge the anonymous referee for her/his valuable comments, that helped improving the quality of the manuscript.

\section{References}

Altavilla, G., Fiorentino, G., Marconi, M., et al. 2004, MNRAS, 349, 1344 Astier, P., Guy, J., Regnault, N., et al. 2006, A\&A, 447, 31 Benetti, S., Cappellaro, E., Mazzali, P. A., et al. 2005, ApJ, 623, 1011 Bessell, M. S. 1990, PASP, 102, 1181

Cardelli, J. A., Clayton, G. C., \& Mathis, J. S. 1989, ApJ, 345, 245 Conley, A., Carlberg, R. G., Guy, J., et al. 2007, ApJ, 664, L13

Cowan, G. 1998, Statistical Data Analysis (Oxford: Clarendon Press) Elias-Rosa, N., Benetti, S., Cappellaro, E., et al. 2006, MNRAS, 369, 1880 Elias-Rosa, N., Benetti, S., Turatto, M., et al. 2008, MNRAS, 384, 107 Ellis, R. S., Sullivan, M., Nugent, P. E., et al. 2007, ArXiv e-prints, 710 Garavini, G., Folatelli, G., Nobili, S., et al. 2007, A\&A, 470, 411 Goldhaber, G., Groom, D. E., Kim, A., et al. 2001, ApJ, 558, 359 Goudfrooij, P., de Jong, T., Hansen, L., \& Norgaard-Nielsen, H. U. 1994, MNRAS, 271, 833

Guy, J., Astier, P., Nobili, S., Regnault, N., \& Pain, R. 2005, A\&A, 443, 781
Guy, J., Astier, P., Baumont, S., et al. 2007, A\&A, 466, 11 Hamuy, M., Phillips, M. M., Suntzeff, N. B., et al. 1996, AJ, 112, 2398 Hoeflich, P., Wheeler, J. C., \& Thielemann, F. K. 1998, ApJ, 495, 617

Hsiao, E. Y., Conley, A., Howell, D. A., et al. 2007, ApJ, 663, 1187 Jha, S., Kirshner, R. P., Challis, P., et al. 2006, AJ, 131, 527 Jha, S., Riess, A. G., \& Kirshner, R. P. 2007, ApJ, 659, 122 Kasen, D., \& Woosley, S. E. 2007, ApJ, 656, 661

Kim, A., Goobar, A., \& Perlmutter, S. 1996, PASP, 108, 190 Knop, R. A., Aldering, G., Amanullah, R., et al. 2003, ApJ, 598, 102 Krisciunas, K., Hastings, N. C., Loomis, K., et al. 2000, ApJ, 539, 658 Krisciunas, K., Suntzeff, N. B., Candia, P., et al. 2003, AJ, 125, 166 Krisciunas, K., Phillips, M. M., Suntzeff, N. B., et al. 2004a, AJ, 127, 1664 Krisciunas, K., Suntzeff, N. B., Phillips, M. M., et al. 2004b, AJ, 128, 3034 Lentz, E. J., Baron, E., Branch, D., Hauschildt, P. H., \& Nugent, P. E. 2000, ApJ, 530,966

Nobili, S., Goobar, A., Knop, R., \& Nugent, P. 2003, A\&A, 404, 901

Nordin, J., Goobar, A., \& Jonsson, J. 2008, ArXiv e-prints, 801

Nugent, P., Phillips, M., Baron, E., Branch, D., \& Hauschildt, P. 1995, ApJ, 455, L147

Nugent, P., Kim, A., \& Perlmutter, S. 2002, PASP, 114, 803

O’Donnell, J. E. 1994, ApJ, 422, 158

Ostman, L., Goobar, A., \& Mortsell, E. 2007, ArXiv e-prints, 711

Pastorello, A., Taubenberger, S., Elias-Rosa, N., et al. 2007, MNRAS, 161

Patat, F., Benetti, S., Cappellaro, E., \& Turatto, M. 2006, MNRAS, 369, 1949

Patil, M. K., Pandey, S. K., Sahu, D. K., \& Kembhavi, A. 2007, A\&A, 461, 103

Perlmutter, S., Gabi, S., Goldhaber, G., et al. 1997, ApJ, 483, 565

Perlmutter, S., Aldering, G., Goldhaber, G., et al. 1999, ApJ, 517, 565

Phillips, M. M., Lira, P., Suntzeff, N. B., et al. 1999, AJ, 118, 1766

Pignata, G., Patat, F., Benetti, S., et al. 2004, MNRAS, 355, 178

Riess, A. G., Press, W. H., \& Kirshner, R. P. 1996, ApJ, 473, 588

Riess, A. G., Filippenko, A. V., Challis, P., et al. 1998, AJ, 116, 1009

Stanishev, V., Goobar, A., Benetti, S., et al. 2007, A\&A, 469, 645

Suntzeff, N. B. 2000, in Amer. Inst. Phys. Conf. Ser. 522, ed. S. S. Holt, \& W. W. Zhang, 65

Tripp, R. 1998, A\&A, 331, 815

Tripp, R., \& Branch, D. 1999, ApJ, 525, 209

Vinkó, J., Bíró, I. B., Csák, B., et al. 2003, A\&A, 397, 115

Wang, L. 2005, ApJ, 635, L33

Wang, X., Li, W., Filippenko, A. V., et al. 2007, arXiv e-prints, 711

Wells, L. A., Phillips, M. M., Suntzeff, B., et al. 1994, AJ, 108, 2233

Wood-Vasey, W. M., Miknaitis, G., Stubbs, C. W., et al. 2007, arXiv Astrophysics e-prints 